\title{
Note on the Magnetic Moment of the Nitrogen Nucleus
}

\author{
R. F. BACHER, * University of Michigan
}

(Received April 26, 1933)

\begin{abstract}
Three lines of the group $2 s 2 p^{4} P-2 s^{2} 2 p^{2} 4 p^{4} D$ of $\mathrm{N} \mathrm{I}$ have been examined for hyperfine structure. They were found to be single when examined with a Fabry-Perot interferometer, using a variety of plate separations. The line widths of two of the lines were measured. The absence of hyperfine structure is attributed to the small magnetic moment $(\mu)$ of the nitrogen nucleus. It is shown that, by using the results of Goudsmit, the measured line widths
\end{abstract}

lead to an unusually low magnetic moment for the nitrogen nucleus, $\mu \leqq 0.2 \mathrm{eh} / 4 \pi M c$. Under certain assumptions about the structure of the nitrogen nucleus, this low value of the magnetic moment leads to the conclusion that the neutron has a magnetic moment in the opposite direction from its mechanical moment and about one proton magneton in magnitude.

$\mathbf{I}^{\mathrm{T}}$ $\mathrm{T}$ is known from the alternation of intensities of the lines of the $\mathrm{N}_{2}+$ bands that the nitrogen nucleus has a mechanical moment of $1 \times h / 2 \pi$. None of the stronger lines of the atomic spectrum which have been investigated, ${ }^{1}$ however, have been observed to show any hyperfine structure. This absence of hyperfine structure may be due to one or both of the following reasons. (1) The level separations of initial and final states may be much the same and the strong lines may, therefore, all fall together. (2) The level separations themselves may be small.

In order to eliminate (1) as a possible cause of the absence of hyperfine structure, combinations of the $2 s 2 p^{4}$ configuration (expected from the separation factors (3) to have hyperfine structure separations roughly 25 times as large as other configurations of $\mathrm{N} \mathrm{I}$ ) with the $2 s^{2} 2 p^{2} 4 p$ were examined. Three lines in particular of this group were studied: $\lambda 5329\left(2 s \quad 2 p^{4}{ }^{4} P_{2 \frac{1}{2}}-2 s^{2} 2 p^{2} 4 p\right.$ $\left.{ }^{4} D_{3 \frac{1}{2}}\right), \lambda 5357$ (2s $\left.2 p^{4}{ }^{4} P_{1 \frac{1}{2}}-2 s^{2} 2 p^{2} 4 p^{4} D_{2 \frac{1}{2}}\right)$ and $\lambda 5373$ (2s $2 p^{4}{ }^{4} P_{\frac{1}{3}}-2 s^{2} 2 p^{2} 4 p^{4} D_{1 \frac{1}{3}}$ ). Of these the first two were more advantageous for study because of their greater intensity and comparative isolation. These lines were found to be excited with considerable intensity in the positive column of a condensed discharge. The discharge tube was designed with a section of the positive column in a $\mathrm{U}$ so that it could be cooled with liquid air and the tube was operated while helium containing a small quantity of nitrogen was circulated through it. The intensity of the nitrogen lines

\footnotetext{
* Alfred Lloyd Fellow.

${ }^{1}$ Gibbs and Kruger, Phys. Rev. 37, 1702 (1931).
}

was considerably increased when the tube was cooled with liquid air. The spectrum was examined with a Fabry-Perot type interferometer with various plate separations from $5 \mathrm{~mm}$ to 29.5 $\mathrm{mm}$ used in conjunction with a spectrograph of the Hilger $\mathrm{E}_{1}$ type (glass).

None of the lines mentioned above showed any structure even when they were examined under the high resolution of the $29.5 \mathrm{~mm}$ plate separation. The widths of the fringes of the lines $\lambda 5329$ and $\lambda 5357$ were determined from measurements on plates taken with the latter separation and were $0.055 \mathrm{~cm}^{-1}$ and $0.053 \mathrm{~cm}^{-1}$ respectively. In addition the fringes shaded off equally on both sides, while if there were any hyperfine structure as large as $0.055 \mathrm{~cm}^{-1}$, an unsymmetrical broadening would be expected since a separation as large as the measured width is well within the practical resolving power of the instrument.

In order to draw any conclusions from the absence of hyperfine structure in these lines and from the measured fringe widths, it is necessary to discuss the size of the expected hyperfine structure. The hyperfine structure separation of an atomic level of quantum number $J$ may be written in the form (1).

$$
d \nu=\frac{1}{2} A\{F(F+1)-J(J+1)-I(I+1)\} .
$$

$I$ is the nuclear moment in units $h / 2 \pi, A$, the interval factor, is a constant which depends on the atomic state and on the magnetic moment of the nucleus, and $F$ is the hyperfine quantum number. In case the hyperfine structure is attributed to the interaction of a single electron, 1001 
the interval factor (called $a$ in this case) may be written $^{2}$ as in (2).

$$
a=\frac{R \alpha^{2} Z_{i} Z_{0}^{2}}{n_{0}^{3}\left(l+\frac{1}{2}\right) j(j+1)} \frac{g(I)}{1838} .
$$

$g(I)$ is the nuclear $g$-factor, i.e., the ratio of magnetic to mechanical moment for the nucleus, $n_{0}$ is the effective total quantum number for the electron, and $Z_{i}$ and $Z_{0}$ are the effective nuclear charge near the nucleus and away from the nucleus, respectively. Using values of $n_{0}$ from the known values of the energy states and the proper values of $l$ and $j$ and reasonable values of $Z_{i}$ and $Z_{0}$, one may find the following:

$$
\begin{aligned}
a(2 s) & =0.23 g(I) \mathrm{cm}^{-1}, \\
a(3 s) & =0.01 g(I) \mathrm{cm}^{-1}, \\
a\left(2 p_{\frac{1}{3}}\right) & =0.014 g(I) \mathrm{cm}^{-1}, \\
a\left(2 p_{13}\right) & =0.003 g(I) \mathrm{cm}^{-1} .
\end{aligned}
$$

The lower two are actually obtained ${ }^{2}$ from a knowledge of the multiplet separation in $2 s 2 p^{4}$ ${ }^{4} P$ of $\mathrm{N} \mathrm{I}$. From these values it may immediately be seen that the effects of the others may be neglected in comparison to that of the $2 s$ electron.

When it is known that the hyperfine structure may be attributed largely to the $2 s$ electron, the separation factors for the three levels $2 s 2 p^{4}$ ${ }^{4} P_{2 \frac{1}{2}, 1 \frac{1}{2}, \frac{1}{3}}$ may easily be found. ${ }^{3}$

$$
\begin{gathered}
A\left(2 \frac{1}{2}\right)=0.046 g(I) \mathrm{cm}^{-1}, \quad A\left(1 \frac{1}{2}\right)=0.056 g(I) \mathrm{cm}^{-1}, \\
A\left(\frac{1}{2}\right)=0.127 g(I) \mathrm{cm}^{-1} .
\end{gathered}
$$

Fig. 1 shows the expected level separations and the resultant line structures and intensities of $\lambda 5329$ and $\lambda 5357$. The separations are all given in terms of $g(I)$ which for nitrogen is numerically the same as the magnetic moment in proton units since $I=1(\mu=g(I) \times I)$. The expected hyperfine structures are both of the flag type, shading somewhat in intensity from one side to the other. The observed fringes showed no such shading. Using the measured fringe widths as upper limits for the widths of possible hyperfine structure one may find that $g(I) \leqq 0.2$ and hence

$$
\mu \leqq 0.2 e h / 4 \pi M c .
$$

\footnotetext{
${ }^{2}$ Goudsmit, Phys. Rev. 43, 636 (1933).

${ }^{3}$ Goudsmit and Bacher, Phys. Rev. 34, 1501 (1929).
}
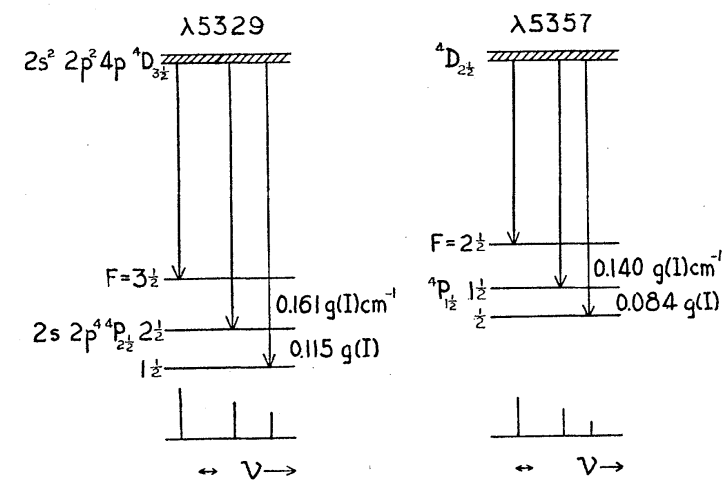

FIG. 1. Level separations and resultant hyperfine structure pattern of $\lambda 5329$ and $\lambda 5357$ of N I given in terms of $g(I)$, as expected theoretically. The small double arrows at the bottom give the measured line widths on the same scale as the expected separations with $g(I)=1$.

It is thus indicated that the magnetic moment of the nitrogen nucleus is considerably smaller than other nuclear magnetic moments ${ }^{2}$ known at present since these range from 0.55 to 5.4 "proton magnetons." It is particularly interesting to see what such a small value of the nuclear magnetic moment means if one considers the structure of the nitrogen nucleus. If one assumes (a) that light nuclei contain as many $\alpha$-particles as possible (or their equivalent in closed groups) then the most probable structure of the nitrogen nucleus is the following:

$$
3 \alpha+1 \text { proton }+1 \text { neutron. }
$$

In addition, it is assumed that $(b)$ the nuclear spin is the resultant of the spins of the particles ${ }^{4}$ in the nucleus and that $(c)$ in analogy to the electron the proton has a magnetic moment $e h / 4 \pi M c$, one "proton magneton." The mechanical moment of $1 \times h / 2 \pi$ is accounted for by the proton and the neutron both having spins ${ }^{5} \frac{1}{2} \times h / 2 \pi$ and having them oriented in the same direction. It is not possible, however, to get $\mu \leqq 0.2$ unless the neutron has its magnetic moment in the opposite direction from its mechanical moment and of a magnitude about one proton magneton. It must be recognized that this conclusion is dependent on hypotheses $(a),(b)$ and $(c)$ and while they seem reasonable at the present moment they are nevertheless speculative.

\footnotetext{
${ }^{4}$ The possible rôle of the positron as a nuclear con: stituent possessing spin is not considered.

${ }^{5}$ Bacher and Condon, Phys. Rev. 41, 683 (1932).
} 